\title{
BMJ Open Quality of life in elderly ICU survivors before the COVID-19 pandemic: a systematic review and meta-analysis of cohort studies
}

\author{
Kevin Ariyo (D) , ${ }^{1}$ Sergio Canestrini, ${ }^{2}$ Anthony S David, ${ }^{3}$ Alex Ruck Keene, ${ }^{1,4}$ \\ Sebastian Wolfrum, ${ }^{5,6}$ Gareth Owen ${ }^{1}$
}

To cite: Ariyo $\mathrm{K}$, Canestrini S, David AS, et al. Quality of life in elderly ICU survivors before the COVID-19 pandemic: a systematic review and meta-analysis of cohort studies. BMJ Open 2021;11:e045086. doi:10.1136/ bmjopen-2020-045086

- Prepublication history and additional supplemental material for this paper are available online. To view these files, please visit the journal online (http://dx.doi.org/10.1136/ bmjopen-2020-045086).

Received 21 September 2020 Accepted 20 July 2021

Check for updates

(C) Author(s) (or their employer(s)) 2021. Re-use permitted under CC BY. Published by BMJ.

For numbered affiliations see end of article.

Correspondence to

Kevin Ariyo;

kevin.ariyo@kcl.ac.uk

\section{ABSTRACT}

Objectives The influence of age on intensive care unit (ICU) decision-making is complex, and it is unclear if it is based on expected subjective or objective patient outcomes. To address recent concerns over age-based ICU decision-making, we explored patient-assessed quality of life (QoL) in ICU survivors before the COVID-19 pandemic. Design A systematic review and meta-analysis of cohort studies published between January 2000 and April 2020, of elderly patients admitted to ICUs.

Primary and secondary outcome measures We extracted data on self-reported QoL (EQ-5D composite score), demographic and clinical variables. Using a random-effect meta-analysis, we then compared QoL scores at follow-up to scores either before admission, agematched population controls or younger ICU survivors. We conducted sensitivity analyses to study heterogeneity and bias and a qualitative synthesis of subscores.

Results We identified 2536 studies and included 22 for qualitative synthesis and 18 for meta-analysis $(n=2326$ elderly survivors). Elderly survivors' QoL was significantly worse than younger ICU survivors, with a small-to-medium effect size $(\mathrm{d}=0.35(-0.53$ and -0.16$))$. Elderly survivors' QoL was also significantly greater when measured slightly before ICU, compared with follow-up, with a small effect size $(\mathrm{d}=0.26(-0.44$ and -0.08$))$. Finally, their $\mathrm{Q}$ L was also marginally significantly worse than age-matched community controls, also with a small effect size $(\mathrm{d}=0.21$ $(-0.43$ and 0.00$))$. Mortality rates and length of follow-up partly explained heterogeneity. Reductions in QoL seemed primarily due to physical health, rather than mental health items.

Conclusions The results suggest that the proportionality of age as a determinant of ICU resource allocation should be kept under close review and that subjective QoL outcomes should inform person-centred decision -aking in elderly ICU patients.

PROSPERO registration number CRD42020181181.

\section{INTRODUCTION}

The influence that age should have on intensive care decision-making has been debated across policy and clinical practice. ${ }^{2}$ Age associates (inversely) with the probability of intensive care unit (ICU) survival and length
Strengths and limitations of this study

- To our knowledge, this is the first systematic review and meta-analysis to explore quality of life (QoL) outcomes in elderly intensive care unit survivors and to explore sources of variation between these studies.

- To ensure consistency and policy relevance, we only included one type of measure within the metaanalysis (EQ-5D).

- With our large sample, we could estimate the population QoL with reasonable precision, as evidenced by narrow Cls.

- Wide prediction intervals suggest that our results should not be used to make individual-level predictions. Our sample had a mixture of conditions, and because data were reported inconsistently and often at study level, it is difficult to generalise to specific clinical groups, including patients with COVID-19.

of life after ICU, ${ }^{34}$ outcomes generally considered to be relevant to resource allocation. ${ }^{2}$ However, age is also a protected characteristic in several jurisdictions, and in England and Wales, resource allocation based on age must be a "proportionate means of achieving a legitimate aim', if it is not to be contrary to the Equality Act (2010).

For elderly patients for whom admission to ICU is clinically appropriate, an important part of person-centred decision-making is for them, or their families, to be given information about the likely outcome of admission. Patients may seek to integrate survival and biomedical outcomes with subjective outcomes, including quality of life (QoL). Subjective QoL in elderly ICU survivors has been studied less frequently than these objective measures. ${ }^{35}$ This is notable given that subjective QoL (via quality-adjusted life years or QALYs) is very influential in clinical resource allocation (eg, at the National Institute for Health and Care Excellence; NICE). 
Person-centred decision-making requires consideration of patient experience since physician-rated QoL is not always well correlated with patient-rated QoL.

We considered a rapid review to be urgent because age is a strong risk factor for severe COVID-19 infection, ${ }^{6}$ and severe COVID-19 has placed considerable pressure on ICU resource allocation ${ }^{7}$ and is likely to do so in the future. Additionally, some have expressed concerns that elderly adults may have been disproportionately less likely to receive ICU before the pandemic. ${ }^{128-10}$ As health system collapse remains a possibility, this raises the prospect of difficult triage decisions. In particular, services will need to weigh up various ethical positions to decide how important age is to these admission policies. ${ }^{11}$ It is therefore important that older persons' subjective outcomes are better understood.

We conducted a meta-analysis on patient-reported QoL in elderly adults undergoing ICU. Following a systematic review, we addressed three questions:

1. At follow-up, do elderly ICU survivors have better/ worse QoL compared with their scores before ICU?

2. At follow-up, do elderly ICU survivors have better/ worse QoL than age-matched community controls?

3. At follow-up, do elderly ICU survivors have better/ worse QoL than ICU survivors aged under 65?

Determining the effect of illness and ICU on QoL is complicated because QoL is itself influenced by many variables $^{12}$ and some are non-clinical. These influences are too complex to resolve completely, but where possible, we sought to model relevant variables (illness severity, ICU length of stay and mortality rate) as predictors of QoL in elderly ICU survivors at follow-up, compared with controls.

\section{METHODS}

\section{Search strategy}

We searched for English-language journal articles, published between January 2000 and April 2020. Six online bibliographical databases were used: Central, CINAHL, Cochrane Library, EMBASE, MEDLINE and PsycINFO. Our search followed a prepublished PROSPERO protocol.

The search terms focused on intensive care, elderly adults and QoL (see item 6 of the online supplemental appendix). We supplemented this with a forward citation and reference list search based on the eligible articles as well as consultation with experts.

\section{Patient and public involvement}

No patient or public advisers were involved in this project.

\section{Selection criteria}

We undertook study selection using EndNote X9 using a standardised crib sheet. See figure 1 for an overview. The inclusion and exclusion criteria are detailed further in item 6 of the online supplemental appendix.
At the title and abstract level, we identified potentially eligible studies that took place in an ICU and referred to either QoL or elderly adults. Full texts were eligible if (a) all participants underwent ICU; (b) there were at least 20 elderly patients and controls; (c) scores from a validated QoL scale were reported, for a group aged at least 60+, with at least 3-month follow-up review; (d) the follow-up QoL scores were derived from the patient, rather than a professional; and (e) the study reported QoL scores from the same scale for either the same patients before the ICU admission, age-matched community controls or ICU survivors aged under 65 .

Where we could not include potentially eligible studies, due to poor reporting, we contacted study authors for unpublished data. We also considered whether to include studies that focused only on cardio-surgical or neurosurgical patients, given the effects of the diagnostic heterogeneity that characterises the reference population of the studies included in our review (general ICU patients with various conditions). However, none of these studies met the other inclusion criteria.

KA led the study selection at all stages, and a postdoctoral research assistant conducted reliability checks for $50 \%$ of full-text articles. We found nearly perfect inter-rater agreement, as measured by Cohen's kappa $(\mathrm{k}=0.86) .{ }^{13}$ Queries were resolved by GO.

\section{Data extraction}

One reviewer (KA) extracted relevant data from all eligible studies, recording this on a standardised spreadsheet. MK independently extracted data from $10 \%$ of eligible studies, to evaluate consistency. The primary outcome was the QoL composite scores. Secondary variables included demographics, QoL subscale scores, mortality (from ICU to follow-up), illness severity (either the Acute Physiology and Chronic Health Evaluation, APACHE-II; or the Simplified Acute Physiology Score, SAPS-II), length of ICU stay, length of hospital stay, and average follow-up time. When one dataset was used for multiple studies, we included the study with the clearest data reporting.

To ensure consistency, we included only composite scores from the EuroQoL health-related QoL instrument (EQ-5D) within the meta-analysis. Where possible, we also converted the eight subscales of the 36-item Short Form Survey (SF-36) to an EQ-5D Index Score, using an established mapping algorithm. ${ }^{14}$ The remaining studies were included within the qualitative synthesis only.

\section{Data analysis}

We explored the effect of age on EQ-5D composite scores using random-effect meta-analyses. KA conducted the analysis using $\mathrm{R}$ Statistics. We used the restricted maximum likelihood method to calculate the effect sizes (Cohen's d), which were weighted by the inverse of the sampling variance: meaning that studies with higher variance contributed less to the summary effect size. We interpreted these effect sizes using conventional criteria as a guide $(0.2=$ small; $0.5=$ medium; $0.8=$ large $) .{ }^{15}$ We then 


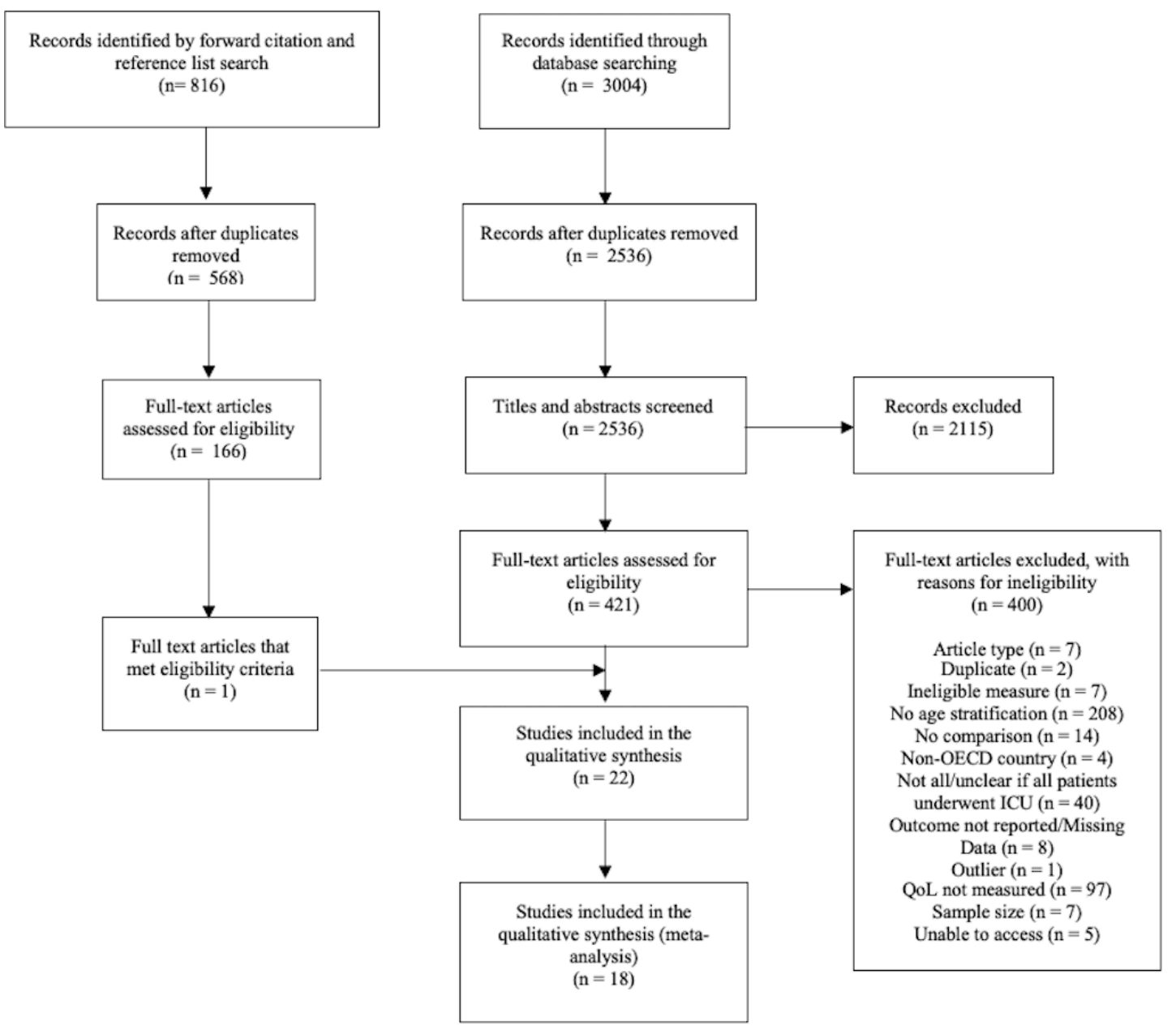

From: Moher D, Liberati A. Tetzlaff J, Altman DG. The PRISMA Group (2009). Preferred Reporting /tems for Systematic Reviews and Meta-

Figure 1 A Preferred Reporting Items for Systematic Reviews and Meta-Analyses flow diagram that outlines the study selection process. ICU, intensive care unit; OECD, Organisation for Economic Co-Operation and Development; QoL, quality of life.

conducted sensitivity analyses for each meta-analysis to assess risk of bias at the study level, including heterogeneity (eg, I ${ }^{2}$ statistic), influential studies (eg, Cook's distance) and publication bias (funnel plots and Egger's test).

To investigate the remaining heterogeneity, we then conducted two secondary analyses: a moderator analysis to explore variation within a specific predictor and a random-effect meta-regression to explore relationships between multiple predictors.

We used several strategies to handle missing data. When the study only reported median values and IQRs, we estimated the mean and SD using conventional formulae. ${ }^{1617}$ When neither the SD nor IQR was reported, we estimated the SD using prognostic imputation. ${ }^{18}$ This calculates the average of observed variances to estimate the missing SD values. We excluded studies with missing data if these methods were inapplicable.

One reviewer (KA) assessed the methodological rigour of the included studies using an 11-item quality checklist (three irrelevant items were excluded) ${ }^{19}$ The criteria were scored as either 2 (complete fulfilment), 1 (partial fulfilment) or 0 (not fulfilled). We then calculated a total score for each study and rated them as either high quality (17/22 or higher), moderate quality (between 10/22 and $16 / 22)$ or low quality (9/22 or lower). Queries were resolved through discussion with GO and SC.

For the qualitative synthesis, we defined a set of criteria for each measure to allocate subscores to either 'mental health' or 'physical health' categories. We then calculated a crude average for subscales within these two categories and weighted them on a scale of 1-100 ( $0=$ minimum QoL; 
$100=$ maximum QoL). As this approach is subjective, we present these findings only as a qualitative supplement.

This study follows methodological guidance from Preferred Reporting Items for Systematic Reviews and Meta-Analyses (see online supplemental appendix).

\section{RESULTS}

\section{Descriptive statistics}

After screening duplicates, the database search revealed 2536 records for title and abstract screening. From these, we reviewed 421 potentially relevant full-text articles for eligibility. Sixteen of these studies met the full criteria and were included in the initial meta-analysis. A further two studies were deemed eligible following a forward citation search and contact with study authors. This led to a total of 18 studies included in the initial meta-analysis $(n=2326$ elderly adults). Eleven of these studies reported age characteristics for the elderly patients $($ mean=79.04), while the others reported the minimum age only.

Most of the studies included both medical and surgical ICU patients ( 15 studies). The remaining studies focused on surgical (two studies) or medical (one study) patients only. A full breakdown of reasons for admissions is available in the online supplemental appendix.

Three types of outcome were included in the metaanalysis. These results compared QoL at follow-up to either pre-ICU scores (five studies), age-matched community controls (ten studies) or younger survivors of ICU (six studies). We provide a full summary in table 1 .

For the qualitative analysis, we identified four further studies. Five different measurement scales were reported: the EuroQoL EQ-5D health-related QoL instrument (EQ-5D Utility Index or Visual Analogue Scale, eleven studies), the short-form medical outcome questionnaire (SF-36, eight studies), the Nottingham Health Profile (one study), the QoL Index (one study) and the WHO QoL instruments (WHOQOL-BREF, one study). SF-36 scores were converted to EQ-5D Index scores for the meta-analysis, while the other measures were excluded (see 'Methods' section).

\section{Meta-analyses}

Table 2 outlines the results of the three meta-analyses. There was a significant difference in EQ-5D composite scores between elderly patients before and after ICU, with a small effect size $(d=-26, p=0.005)$. This suggests that elderly patients may expect a slightly worse QoL at follow-up, compared with their own scores 1 month before ICU.

There was a marginally significant difference in EQ-5D composite scores between elderly ICU survivors and agematched community controls, with a small effect size $(\mathrm{d}=-0.22, \mathrm{p}=0.05)$. These results suggest that QoL may be slightly lower in elderly ICU survivors, relative to community controls.

Elderly ICU survivors (aged over 65) had significantly lower composite scores on the EQ-5D, compared with younger ICU survivors (aged under 65), with a small-tomedium effect size $(\mathrm{d}=-0.33, \mathrm{p}<0.01)$. This suggests that on average, QoL in elderly ICU survivors is slightly worse than younger ICU survivors.

\section{Sensitivity analyses}

We reviewed the impact of influential cases within each analysis. One study was excluded from the community meta-analysis as a substantial outlier and influential result. If the result had not been excluded, the effect size would have been stronger $(\mathrm{d}=-1.97$-ie, a larger difference in QoL favouring younger controls) but non-significant $(\mathrm{p}=0.27)$, mainly due to large heterogeneity $\left(\mathrm{I}^{2}=100 \%\right)$. It is unclear why this study reported substantially outlying results, although the reported SDs were considerably lower than other studies.

After excluding this, one other study was somewhat influential within the community analysis (see online supplemental appendix). This study was retained as we acquired the full dataset, and we can therefore be confident of its reporting accuracy. If this study was excluded, the effect size would have been weaker $(d=-0.13)$ and non-significant (0.010) in the same direction.

We identified no further outliers according to our criteria.

\section{Secondary analyses}

There was moderate-to-large heterogeneity between studies. For significant results, we explored the role of other variables using post hoc subgroup analyses and meta-regressions. These results should be interpreted with caution, due to low sample sizes.

Length of follow-up significantly predicted greater differences in QoL between elderly ICU survivors and patients aged under $65(\mathrm{k}=6, \mathrm{p}<0.001)$. This suggests that elderly survivors may have worse QoL in the long term and comparable QoL in the medium term.

The minimum age of the sample significantly predicted greater differences in QoL between elderly ICU survivors and age-matched community controls $(\mathrm{k}=10, \mathrm{p}=0.02)$. Subgroup analyses revealed that in studies with only very old patients (aged 75-80+), elderly ICU survivors' QoL was no worse than their age-matched community controls $(\mathrm{k}=6, \mathrm{~d}=-0.06, \mathrm{p}>0.05)$. In contrast, when elderly was defined as 65-70+, elderly ICU survivors had much worse QoL than age-matched community controls $(\mathrm{k}=4, \mathrm{~d}=0.45, \mathrm{p}<0.03)$. This suggests that some of the variation was due to age differences in QoL in community controls.

Controlling for these variables reduced heterogeneity between studies by $10 \%$ and $47 \%$, in both cases. No model significantly accounted for variance when the outlier was included in the community analysis.

Neither severity of illness, year of publication nor sex significantly accounted for heterogeneity between the studies, either individually or within a meta-regression ( $>0.05$ ). 


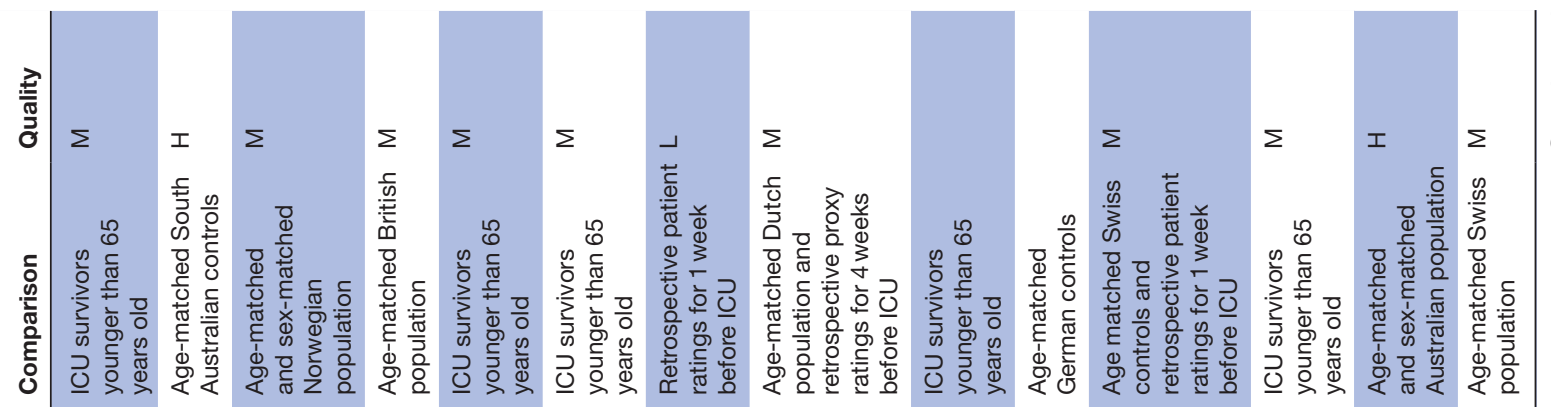

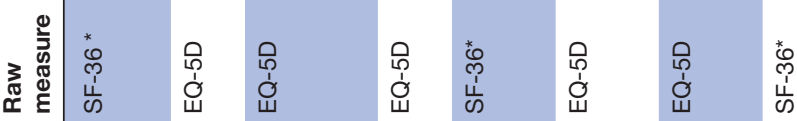

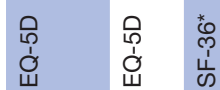

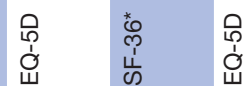

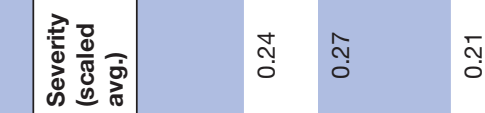

$\stackrel{\infty}{\stackrel{\infty}{0}} \stackrel{\stackrel{0}{0}}{0}$

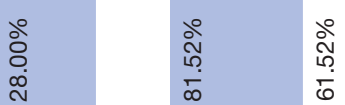

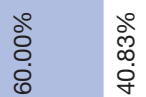

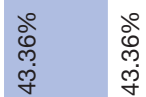

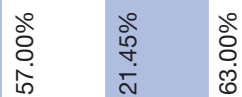

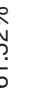

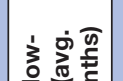

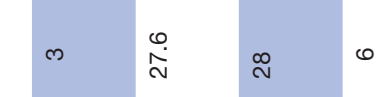




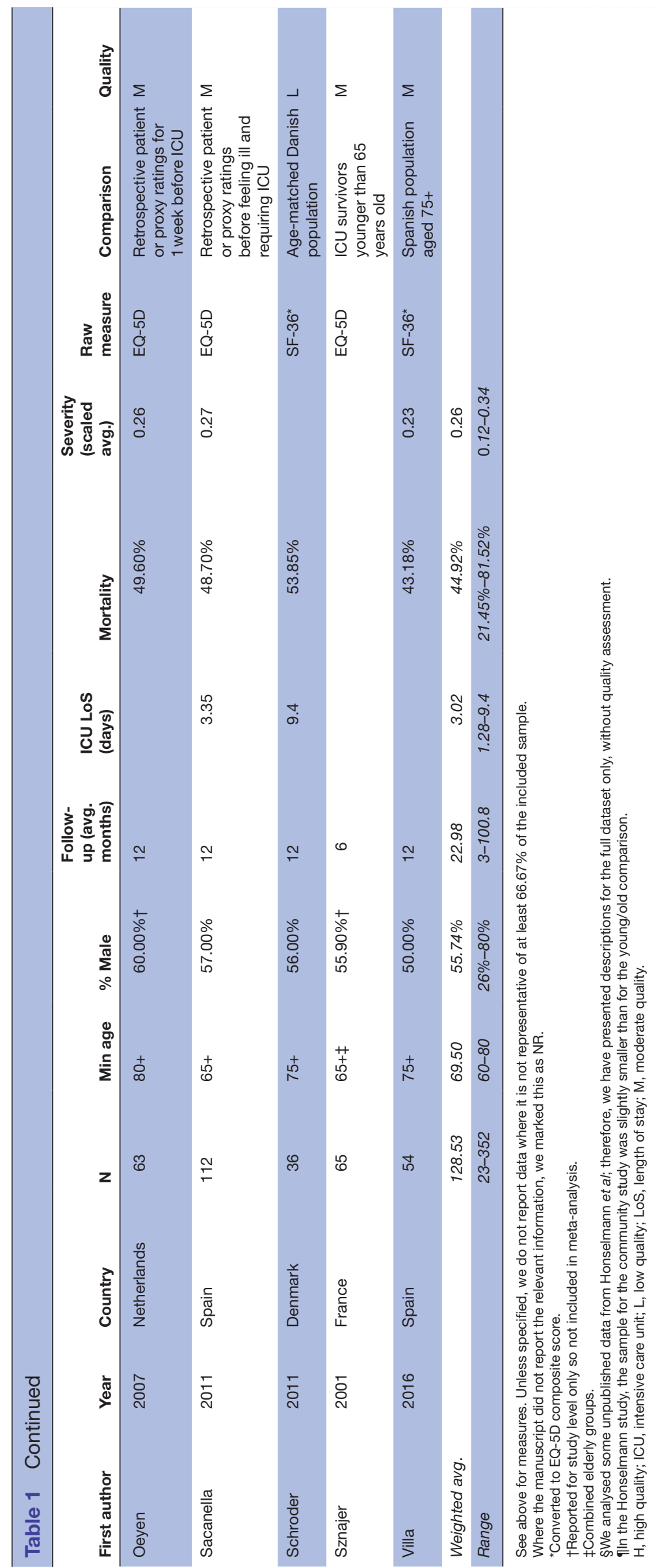

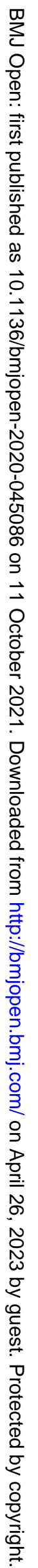


Table 2 A summary of effect sizes, Cls, prediction intervals (PIs), significance and heterogeneity for each meta-analysis

\begin{tabular}{lclllll}
\hline Comparison & k & Cohen's d & $95 \% \mathbf{C l}$ & $\mathbf{9 5 \%} \mathbf{P I}$ & $\mathbf{P}$ & $\mathbf{1}^{\mathbf{2}}$ \\
\hline Pre-ICU scores & 5 & -0.26 & -0.44 to -0.08 & $-0.58,0.07$ & 0.005 & $45.50 \%$ \\
Community & 10 & -0.22 & -0.43 to 0.00 & $-0.88,0.45$ & 0.053 & $87.88 \%$ \\
Under 65 & 6 & -0.35 & -0.53 to -0.16 & $-0.83,0.18$ & 0.000 & $81.93 \%$ \\
\hline
\end{tabular}

$\mathrm{I}^{2}$, between study heterogeneity; $\mathrm{k}$, number of independent samples.

\section{Risk of bias}

We found no evidence for publication bias for the community or pre-ICU meta-analyses, from either funnel plots or Egger's test (all p>0.05). Most studies had a moderate degree of methodological quality $(13 / 17)$. We had insufficient power to explore the effect of study quality on quantitative outcomes.

\section{Qualitative synthesis}

To compare different aspects of QoL, we categorised the subscales into either mental or physical health QoL and calculated a scaled average to enable comparisons (see table 3). Sixteen out of twenty-two studies reported the subscales for both conditions. Our estimates suggest that elderly ICU survivors reported higher average scores on mental health items $($ mean $=57.08 / 100)$ than physical health items $($ mean $=47.12 / 100)$. Trends in physical health scores compared less favourably to age-matched community controls than did mental health scores (mean differences $=-5.23$ and -1.71 , respectively). Trends in physical health scores were also lower in comparison to younger ICU controls (mean difference $=-2.63$ ), whereas mental health scores were higher (mean difference=2.65).

\section{DISCUSSION}

This review has systematically evaluated the literature on QoL for elderly ICU survivors in the medium-to-long term, using EQ-5D composite scores. To our knowledge, this is the first meta-analysis to address this issue. We found evidence that elderly patients who survive ICU can be expected to have slightly worse QoL, compared with younger survivors. To a lesser extent, they may also have worse QoL compared with age-matched community controls and compared with their own QoL up to 1 month before ICU. The wide prediction intervals also suggest that age differences can vary considerably in either direction.

\section{Strengths in relation to the literature}

For the meta-analysis, we identified 2326 elderly ICU survivors within an international sample of 18 cohort studies. We only included recent studies that used validated QoL measures, and we rated most studies as having moderate or higher methodological quality. By pooling these samples using rigorous methods, we have been able to overcome several methodological limitations associated with generalising from individual studies, including small samples, choice of analysis and site selection bias.
Our sensitivity analyses showed that the remaining heterogeneity was partly due to conceptually relevant variables. Given the relatively small literature, these methods ensure that valid, transparent results inform policy and clinical practice decisions.

Although contested, previous reviews have generally concluded that age alone is not a suitable determinant of potential benefit from ICU, especially for survivors. ${ }^{352021}$ The present study supports these conclusions, although the differences compared with younger ICU survivors (and, to a lesser extent, community samples) are still noteworthy. Decisions on whether to admit patients can be extremely difficult for all involved, with seriously ill elderly people over-represented among the most contentious cases. ${ }^{22}$ These challenges are amplified further when healthcare resources are under pressure, such as during the COVID-19 pandemic.

The age-QoL associations we have found may be explained by intermediary variables. Some research suggests that frailty may best explain age differences in QoL following ICU $^{523}$ and clinical outcome in patients with COVID-19. ${ }^{24}$ Frailty is a more integrative approach to conceptualising ageing, but it was not reported within the eligible studies. We would also recommend a metaanalysis of individual patient data for patients with COVID-19, to further stratify clinical variables of interest, including frailty, and to better predict QoL outcomes.

Health economic analysis of ICU in the elderly based on QALYs may be informative when it comes to resource allocation policies, but we have found few such analyses and no explicit polices based on them. They will have to grapple with the controversial notion that everyone is entitled to a 'normal' span of health or 'a fair innings'. ${ }^{25}{ }^{26}$ Given the presumption that a sizeable proportion of elderly survivors will enjoy a good QoL, it is crucial that holistic, person-centred decision-making is not crowded out by survival statistics or anticipatory triage. If triage was to become necessary on the front line, we would advise against weighing age too heavily and rather taking more account of frailty after appropriate consultations.

On average, QoL scores gradually decline with age at approximately 0.5 points per year on the CASP-19 (range $0-57$ ) with a modestly accelerated decrease with older age (>85 years). ${ }^{4}$ It is relevant to consider whether change in QoL in the elderly is primarily due to physical health and mental health components. We were unable to incorporate physical and mental subscores into the meta-analysis 


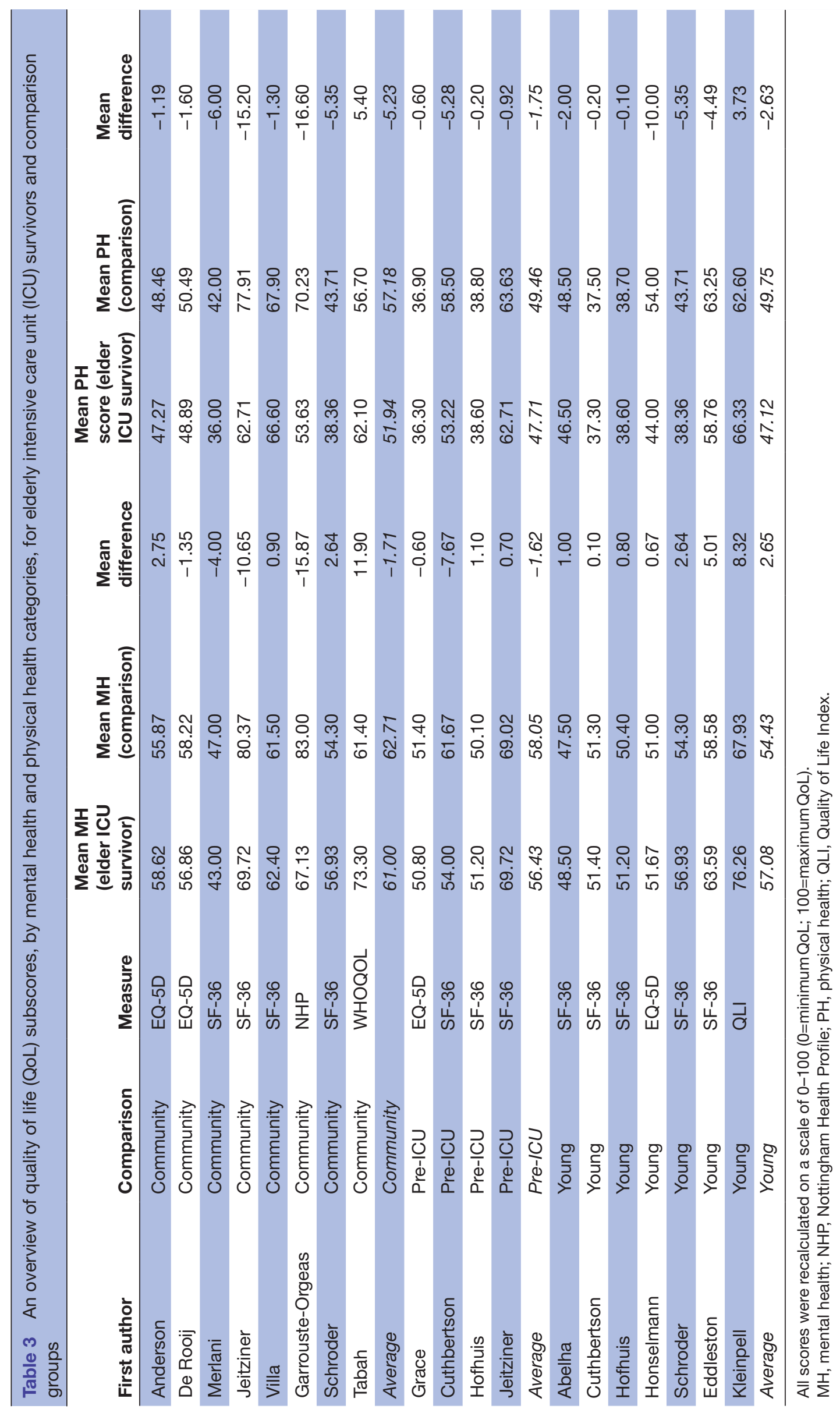


due to differences in the levels of data between measures, so we performed a qualitative synthesis. This suggested that for elderly ICU survivors, mental health questionnaire items were relatively unaffected. The small literature on older adults also suggests relatively low rates of anxiety ${ }^{27}$ and depressive disorders, ${ }^{28} 29$ although potentially high rates of post-traumatic stress. ${ }^{30}$ Further mental health data are needed, as some preliminary reports suggest high rates of post-traumatic stress in ICU patients with COVID-19. ${ }^{31}{ }^{32}$ Our results may serve as a baseline to compare mental and physical health outcomes between COVID-19 and non-COVID-19 survivors.

\section{Limitations}

The primary limitation is the small number of eligible studies for each analysis. To maximise the sample, we included some studies with a small amount of missing data and used validated methods to estimate the mean or the SD from the reported statistics. We argue that these approaches are justified as, based on central limit theorem, we expect the larger sample sizes to produce a better estimate of population variance. ${ }^{33}$ For balance, we have also provided a comprehensive overview of our sensitivity analyses to assess risk of bias (see online supplemental appendix). These demonstrate that although our decisions reduced bias, most did not change our interpretation of the effects.

Another potential limitation of the meta-analysis is the focus on long-term ICU survivors, as reported mortality rates were as high as $80 \%$ at follow-up. We argue that a substantial 'healthy survivor' effect on QoL is unlikely because survival and QoL have different pathophysiological determinants. We also did not find any evidence of better QoL for elderly patients in studies with high mortality rates. Nevertheless, our results clearly extend only to ICU survivors, rather than prospective ICU patients.

Our results may also be prone to other selection biases. Compared with younger adults, unhealthy elderly adults might be less likely to be admitted into the ICU, ${ }^{22} 34$ to survive ICU treatment (possibly in part due to decisions around life-saving treatment ${ }^{35}$ and to survive until follow-up. It was also unclear how many patients had preexisting cognitive impairments where QoL measurement is more complex, although there was no indication that the proportion was large. Without further data on contextual variables, we would caution wider generalisations to all elderly ICU patients. Nonetheless, these results imply that at least some elderly ICU patients will have a relatively good QoL in the medium-to-long term.

In particular, no patients with COVID-19 were included in the sample. COVID-19 pneumonitis has a specific pathophysiology that does not lead to a 'typical' acute respiratory syndrome, and this can require a relatively high degree of multisystemic involvement. Future studies will need to consider elderly COVID-19 survivors, who often require a relatively lengthy period of ICU treatment and post-ICU rehabilitation, especially if unvaccinated.
We were unable to assess QoL as rigorously as we would have liked. This was partly because studies varied in their definitions of 'old age'. Most of the eligible studies defined this as $65+$, following the WHO definition. ${ }^{36}$ However, patients aged $65+$ currently account for roughly half of all ICU admissions. ${ }^{37}$ It is therefore likely that a higher threshold would be more relevant to investigate age-related syndromes. A consensus on what should count as 'very old' would help data collection, analysis and interpretation within this field.

The pre-ICU scores were determined by retrospective ratings from discharged patients or proxies. This is usual practice, but the reliability of proxies is contested. ${ }^{38} 39$

Ideally, we would have analysed differences in QoL change scores between younger and elderly ICU survivors, at multiple time points from before ICU to follow-up.

Finally, we observed moderate-to-high levels of heterogeneity between studies, which limits the generalisability of the results. We found that much of this variation may have been due to mortality rates and length of time post discharge, which supports the view that age alone is not a strong predictor of QoL outcome. We also tried to ensure consistency of measurement by using a mapping function between SF-36 scores and EQ-5D scores, which is a common approach within NICE guidelines. ${ }^{14} 40$

\section{CONCLUSION}

Our study reports the first known meta-analysis of QoL in elderly patients following ICU. We report that on average, elderly survivors of ICU have slightly worse QoL compared with younger ICU survivors, based on physical rather than mental health. To a lesser extent, they may also have worse QoL compared with their own scores before ICU and compared with their community peers. These findings add rigour to the current literature and should inform debates around population-level resource allocation and person-centred intensive care decisionmaking during the current COVID-19 pandemic and after.

\section{Author affiliations}

${ }^{1}$ Department of Psychological Medicine, King's College London, London, UK ${ }^{2}$ Critical Care, King's College Hospital NHS Foundation Trust, London, UK ${ }^{3}$ Institute of Mental Health, UCL, London, UK

${ }^{4}$ Dickson Poon School of Law, King's College London, London, UK

${ }^{5}$ Medical Clinic II, Cardiology/Angiology/Intensive Care Medicine, University Hospital Schleswig Holstein, Lübeck, Germany

${ }^{6}$ Department of Emergency Medicine, University Hospital Schleswig Holstein, Lübeck, Germany

Acknowledgements We are grateful to Margot Kuylen for her contributions to the reliability assessment and to John Brazier for advising on the SF-36 to EQ-5D mapping function.

Contributors KA led at each stage of the project, including drafting the document. $\mathrm{KA}$ also acts as guarantor of the study. G0 was the primary supervisor on the project, jointly formulated the research questions, led on writing the introduction section and made substantial contributions to all aspects of the study. SC advised on the initial protocol and provided critical revisions from an intensivist perspective. ASD and ARK provided additional supervision and critical revisions. SW also contributed to data collection and analysis, by providing previously unpublished 
data, and critical revisions. The manuscript is a transparent account of the study being reported and adheres to PRISMA reporting guidelines. All listed authors have approved for the manuscript to be published in its current format and meet all the ICMJE criteria for authorship. The authors agree to be accountable for the contents of the paper and are jointly responsible for ensuring that all queries related to the accuracy or integrity of the project are investigated and resolved.

Funding Supported by the Mental Health and Justice Project, led by G0, which is funded by a grant from the Wellcome Trust (203376/2/16/Z).

Competing interests ARK is an adviser on the Faculty of Intensive Care Medicine's Legal and Ethical Policy Unit.

Patient consent for publication Not required.

Provenance and peer review Not commissioned; externally peer reviewed.

Data availability statement Data are available upon reasonable request. All data relevant to the study are included in the article or uploaded as supplementary information. The datasets generated and analysed during the current study are included in this published article and its supplementary information files. Any data queries may also be directed to the corresponding author on reasonable request.

Supplemental material This content has been supplied by the author(s). It has not been vetted by BMJ Publishing Group Limited (BMJ) and may not have been peer-reviewed. Any opinions or recommendations discussed are solely those of the author(s) and are not endorsed by BMJ. BMJ disclaims all liability and responsibility arising from any reliance placed on the content. Where the content includes any translated material, BMJ does not warrant the accuracy and reliability of the translations (including but not limited to local regulations, clinical guidelines, terminology, drug names and drug dosages), and is not responsible for any error and/or omissions arising from translation and adaptation or otherwise.

Open access This is an open access article distributed in accordance with the Creative Commons Attribution 4.0 Unported (CC BY 4.0) license, which permits others to copy, redistribute, remix, transform and build upon this work for any purpose, provided the original work is properly cited, a link to the licence is given, and indication of whether changes were made. See: https://creativecommons.org/ licenses/by/4.0/.

\section{ORCID iD}

Kevin Ariyo http://orcid.org/0000-0003-0565-2502

\section{REFERENCES}

1 Archard D, Caplan A. Is it wrong to prioritise younger patients with covid-19? BMJ 2020;369:m1509.

2 Savulescu J, Cameron J, Wilkinson D. Equality or utility? Ethics and law of rationing ventilators. $B r J$ Anaesth 2020;125:10-15.

3 Hennessy D, Juzwishin K, Yergens D, et al. Outcomes of elderly survivors of intensive care: a review of the literature. Chest 2005;127:1764-74.

4 Zaninotto P, Falaschetti E, Sacker A. Age trajectories of quality of life among older adults: results from the English longitudinal study of ageing. Qual Life Res 2009;18:1301-9.

5 Conti M, Merlani P, Ricou B. Prognosis and quality of life of elderly patients after intensive care. Swiss Med Wkly 2012;142:w13671.

6 Zhou F, Yu T, Du R, et al. Clinical course and risk factors for mortality of adult inpatients with COVID-19 in Wuhan, China: a retrospective cohort study. Lancet 2020;395:1054-62.

7 Willan J, King AJ, Jeffery K, et al. Challenges for NHS hospitals during covid-19 epidemic. BMJ 2020;368:m1117.

8 de Castro-Hamoy L, de Castro LD. Age matters but it should not be used to discriminate against the elderly in allocating scarce resources in the context of COVID-19. Asian Bioeth Rev 2020:1-10.

9 Haas LEM, de Lange DW, van Dijk D, et al. Should we deny ICU admission to the elderly? ethical considerations in times of COVID-19. Crit Care 2020;24:1-3.

10 Montero-Odasso M, Hogan DB, Lam R, et al. Age alone is not adequate to determine health-care resource allocation during the COVID-19 pandemic. Can Geriatr J 2020;23:152-4.

11 Kuylen MNI, Kim SY, Ruck Keene A, Keene AR, et al. Should age matter in COVID-19 triage? A deliberative study. J Med Ethics 2021;47:291-5

12 Rubenfeld GD. Does the hospital make you older faster? Am J Respir Crit Care Med 2012;185:796-8.
13 Landis JR, Koch GG. The measurement of observer agreement for categorical data. Biometrics 1977;33:159-74.

14 Ara R, Brazier J. Deriving an algorithm to convert the eight mean SF-36 dimension scores into a mean EQ-5D preference-based score from published studies (where patient level data are not available). Value Health 2008;11:1131-43.

15 Cohen J. Statistical power analysis for the behavioral sciences. New York, NY: Academic, 1988.

16 Luo D, Wan X, Liu J, et al. Optimally estimating the sample mean from the sample size, median, mid-range, and/or mid-quartile range. Stat Methods Med Res 2018;27:1785-805.

17 Wan X, Wang W, Liu J, et al. Estimating the sample mean and standard deviation from the sample size, median, range and/or interquartile range. BMC Med Res Methodol 2014;14:135.

18 Ma J, Liu W, Hunter A, et al. Performing meta-analysis with incomplete statistical information in clinical trials. BMC Med Res Methodol 2008;8:56

19 Kmet LM, Cook LS, Lee RC. Standard quality assessment criteria for evaluating primary research papers from a variety of fields, 2004.

20 Chelluri L, Grenvik A, Silverman M. Intensive care for critically ill elderly: mortality, costs, and quality of life. review of the literature. Arch Intern Med 1995;155:1013-22.

21 Wehler M. [Long-term outcome of elderly patients after intensive care treatment]. Med Klin Intensivmed Notfmed 2011;106:29-33.

22 Escher M, Cullati S, Hudelson P, et al. Admission to intensive care: a qualitative study of triage and its determinants. Health Serv Res 2019;54:474-83.

23 Heyland DK, Garland A, Bagshaw SM, et al. Recovery after critical illness in patients aged 80 years or older: a multi-center prospective observational cohort study. Intensive Care Med 2015;41:1911-20.

24 Hewitt J, Carter B, Vilches-Moraga A, et al. The effect of frailty on survival in patients with COVID-19 (COPE): a multicentre, European, observational cohort study. Lancet Public Health 2020;5:e444-51.

25 National Institute for Care Excellence. NICE citizens Council report on age; 2020.

26 Williams A. Intergenerational equity: an exploration of the 'fair innings' argument. Health Econ 1997;6:117-32.

27 Jeitziner M-M, Hamers JPH, Bürgin R, et al. Long-Term consequences of pain, anxiety and agitation for critically ill older patients after an intensive care unit stay. J Clin Nurs 2015;24:2419-28.

28 Broslawski GE, Elkins M, Algus M. Functional abilities of elderly survivors of intensive care. J Am Osteopath Assoc 1995;95:712.

29 Chelluri L, Pinsky MR, Donahoe MP, et al. Long-Term outcome of critically ill elderly patients requiring intensive care. JAMA 1993;269:3119-23.

30 Drews T, Franck M, Radtke FM, et al. Postoperative delirium is an independent risk factor for posttraumatic stress disorder in the elderly patient: a prospective observational study. Eur J Anaesthesiol 2015;32:147-51.

31 Antonis T. Post-Traumatic stress disorder (Ptsd) in patients with Covid 19. Ann Clin Med Case Rep 2021; V:5.

32 de Graaf MA, Antoni ML, Ter Kuile MM, et al. Short-Term outpatient follow-up of COVID-19 patients: a multidisciplinary approach. EClinicalMedicine 2021;32:100731.

33 Weir CJ, Butcher I, Assi V, et al. Dealing with missing standard deviation and mean values in meta-analysis of continuous outcomes: a systematic review. BMC Med Res Methodol 2018;18:25.

34 Garrouste-Orgeas M, Timsit J-F, Montuclard L, et al. DecisionMaking process, outcome, and 1-year quality of life of octogenarians referred for intensive care unit admission. Intensive Care Med 2006;32:1045-51.

35 Ferrand E, Robert R, Ingrand P, et al. Withholding and withdrawal of life support in intensive-care units in France: a prospective survey. French LATAREA group. Lancet 2001;357:9-14.

36 BRUNDTLAND G. Achieving health across the life span. Men Aging and Health1999.

37 Boumendil A, Somme D, Garrouste-Orgeas M, et al. Should elderly patients be admitted to the intensive care unit? Intensive Care Med 2007;33:1252.

38 Capuzzo M, Grasselli C, Carrer S, et al. Quality of life before intensive care admission: agreement between patient and relative assessment. Intensive Care Med 2000;26:1288-95

39 Dinglas VD, Gifford JM, Husain N, et al. Quality of life before intensive care using EQ-5D: patient versus proxy responses. Crit Care Med 2013;41:9.

40 Longworth L, Rowen D. Mapping to obtain EQ-5D utility values for use in NICE health technology assessments. Value Health 2013;16:202-10. 